\title{
ARTICLES
}

\section{Measuring keyboard response delays by comparing keyboard and joystick inputs}

\author{
HIDEMI SHIMIZU \\ Aichi University of Education, Kariya, Aichi, Japan
}

\begin{abstract}
The response characteristics of PC keyboards have to be identified when they are used as response devices in psychological experiments. In the past, the proposed method has been to check the characteristics independently by means of external measurement equipment. However, with the availability of different PC models and the rapid pace of model change, there is an urgent need for the development of convenient and accurate methods of checking. The method proposed here consists of raising the precision of the PC's clock to the microsecond level and using a joystick connected to the MIDI terminal of a sound board to give the PC an independent timing function. Statistical processing of the data provided by this method makes it possible to estimate accurately the keyboard scanning interval time and the average keyboard delay time. The results showed that measured keyboard delay times varied from 11 to $73 \mathrm{msec}$, depending on the keyboard model, with most values being less than $30 \mathrm{msec}$.
\end{abstract}

PC keyboards (keyboards) have often been used as response panels for the collection of reaction time (RT) data, and the drop in PC prices has led to the appearance of many computer classrooms that can be used as quasilaboratories in obtaining data from many people at the same time. However, the models and manufacturers of the PCs vary from one classroom to another, and the equipment is frequently replaced, so it is important to formally evaluate the consistency of the RT data.

However, the RT recorded by the PC can be affected by many sources of variability that are inseparably connected with the use of the PC. The main possible sources are clock resolution, $\mathrm{CPU}$ processing speed, and various interrupt and response characteristics of input/output (I/O) devices, such as keyboards. Many researchers have attempted to control this variability by using programming techniques and the selection of OSs and/or programming languages. In this paper, a new technique for examining the delay times of various keyboards is proposed in order to cope with the rapidly changing $\mathrm{PC}$ environment

Attention should be paid to three delay factors when one uses a keyboard as a response panel. The first is the physical delay time in keypress, or the time elapsed from contact of a key surface to key-on. The second is the variable

The author greatly appreciates the constructive comments of an anonymous reviewer. He is also indebted to the warm encouragement of Ryoji Osaka. Correspondence concerning this article should be addressed to H. Shimizu, Center for Research, Training and Guidance in Educational Practice, Aichi University of Education, 1-Hirosawa, Igaya, Kariya, Aichi 448-8542, Japan (e-mail: hshimizu@ auecc.aichi-edu. ac.jp). delay time at which a key-on signal is detected by keyboard scanning. When the key-on signal occurs just before the scanning signal, the on signal is detected immediately, whereas an on signal just after a scanning signal will not be detected until the next scanning signal (Fay, 2000). The third is the fixed time delay, which consists of the time it takes to convert a key-on signal to a character code, as well as the time it takes the signal to travel to the CPU (Kawamura \& Yoshida, 1995, p. 228). These three factors determine the total mean delay time for the keyboard, whereas both the first and the second factors determine the error variance for the keyboard.

The keyboard response characteristics have to be clarified in order to guarantee the reliability of the data when a keyboard is used to collect data for psychological experiments. Simultaneous comparison of the data value obtained from a keyboard with the value from a universal counter (UC) enables us to estimate the keyboard total mean delay time and the keyboard error variance. Segalowitz and Graves (1990) "strongly recommended that an external measure of the accuracy be made of any timing done with microcomputers, that such accuracy measurements and corrections be reported in published research articles" (p. 286).

Some researchers, however, do not consider the identification of keyboard response characteristics to be crucial. The keyboard error variance is negligible, as compared with the variance in RT time in many research areas (e.g., lexical decision). However, because a new type of keyboard like the USB connectable one has been developed, there is probably doubt as to whether the keyboard error variance can safely be ignored if it is used. In addition, 
correction of data by the keyboard total mean delay time is necessary when one compares the results of the experiments with those of other researchers. However, some research investigating action mechanisms require very high precision timing, and so self-made devices are introduced, instead of a keyboard. Nevertheless, before a full-scale experiment is begun, researchers occasionally want to run preexperiments using many people at the same time in a computer classroom. In such cases, it is helpful to know the keyboard response characteristics.

Of course, the identification of keyboard response characteristics is indispensable for experiments in which the use of a keyboard is unavoidable, as in problems of typing speed (e.g., Pashler, 1994; Verwey \& Dronkert, 1996). In these experiments, one should pay close attention to the error variance by keyboard scanning, because the error variance that is included in the interresponse time (IRT) measurement from one keypress to the next is theoretically twice that of the RT measurement. Although Ulrich and Giray (1989) claimed, when measuring RTs, that PCs with "time resolutions between 10 and 30ms" (p. 12) are still effective in obtaining meaningful conclusions, more precise time resolutions are required in measurement of IRTs.

Even if the need for the identification of the keyboard response characteristics by external measurement equipment is accepted, the remarkable evolution of today's PC hardware has created problems for the identification technique proposed by Graves and Bradley (1987). The first problem is that the integrated PC motherboard and its arrangement make it difficult to insert a probe to get signals from the speaker, which presents the go signals in RT tasks and is placed on the motherboard, behind a steel plate. The second problem is how to select quickly the best available PCs and keyboards and how to identify the keyboard response characteristics, whatever the setting. When possible, it is better not to have to set up a UC, an oscilloscope, or a data recording device to identify keyboard response characteristics.

The method proposed here is to make the PC itself, to which the keyboard is connected, work like a UC. To realize this, a joystick with a delay time of, generally, $1 \mathrm{msec}$ or less is used (Graves \& Bradley, 1987). The PC's built-in clock and the timer measure two real times corresponding to the PC's speaker-on and the joystick-on, respectively. The difference of the two times gives the interval time.

The first purpose of this paper is to investigate, with the use of many PC/keyboard combinations, how far the joystick values agree with external UC values. The second purpose, if use of a joystick instead of a UC is justified, is to identify the keyboard total mean delay time and the keyboard error variance by comparing RT data from a keyboard with that from a joystick, the RT data value being the interval time from speaker-on to keypress (RT condition). The third purpose is to clarify the keyboard error variance in IRT data by making a comparison with the joystick IRT, the IRT data value being the interval time from one keypress to the next (IRT condition).

\section{PRELIMINARY WORK AND OUTLINE OF HARDWARE}

\section{Preliminary Work}

There are two things that have to be done before putting this method into practice. The first is to install a $\mu$ sec order clock on the PC in order to make it function as a UC, which requires (1) development of a highly precise clock and (2) evaluation of its accuracy.

The second is to keep key touch speed as constant as possible, in order to simplify the estimation of the error variance by keyboard scanning. A simple pen shape with a built-in spring (S-Pen; see Figure 1) was made to achieve this, making it possible to strike the target key at a high speed without fear of hitting other keys. The S-Pen enables us to hold down the small variance that accompanies the physical delay time. The physical delay time could vary from one keyboard model to another, but the difference does not seem to be large. Whether the physical keypress variance, apart from the physical mean delay time, is negligible in comparison with the variance by keyboard scanning will be investigated.

\section{Outline of Hardware}

A joystick and a PCI MIDI board supported by MS-DOS were prepared. The reason for our using MS-DOS was to enable us to measure time accurately, which is more difficult in a multitasking OS, such as Windows, Linux, MacOS, or Unix. With a multitasking OS, it takes time for the computer resources to be switched from one task to another, and so there is no guarantee that the resources will be available exactly when needed. In addition, the PCI connection permits faster data transfer than does the serial data transfer of a keyboard.

We made a circuit that made it possible for the action of striking a key with the S-Pen to result in both pushing the A button of the joystick and pressing the key. The bypass of the A button of the joystick was formed with the tip of the S-Pen and the copperfoil's being attached to the surface of a key (see Figure 1). Thus, the two signals from the joystick and the keyboard were simultaneously transferred to a CPU by one key touch with the S-Pen.

Finally, we will describe a set of external measurement equipment and the procedure used to verify the validity of the joystick use.

A UC (used as an external measurement device), a PC (used for automatically collecting data through a GP-IB connected to the UC), and an oscilloscope for monitoring the electric input waveform were prepared.

We connected the leads to the UC's terminal and the speaker terminal on the PC's motherboard. The speaker activation voltage change started the UC, and then the voltage change sent from the joystick stopped the UC. The data that were measured by the UC were automatically recorded on a $\mathrm{PC}$ used for that purpose.

This step can be omitted if the reliability of the joystick's use can be verified. This makes the removal of part of the steel plate protecting the motherboard's back un- 
necessary to obtain speaker activation signals, as well as introduction of a set of expensive external measurement devices. The keyboard response characteristics are identified, instead, by a \$50 MIDI board for MS-DOS and a \$10 joystick, which are needed to run the program developed here. The MS-DOS system included in Windows 9x/Me makes it easy to construct the floppy-based system disk.

In the following, the above-mentioned preliminary first and second steps will be described in further detail before the main argument is presented.

\section{Preliminary Step 1A: Development of a High-Precision Clock}

In order to examine keyboard response characteristics, measurement needs to be possible at the microsecond level, rather than at the millisecond level. Smith and Puckett (1984) have already realized a method of obtaining time information at the microsecond level by using an 8253 timing chip with machine language (a high-quality 8253-compatible model, such as 82C54, may work well). Graves and Bradley (1987) rewrote this method in BASIC in order to measure human RT with an millisecond order of accuracy.

Their ideas were followed and rewritten in C language, obtaining a precision of $\pm(1 / 1,193,180) \mathrm{sec}= \pm 0.8380965$ $\mu \mathrm{sec}$, which was the same as the theoretical value. ${ }^{1}$ The Borland Company's Turbo $\mathrm{C}++$ version $4.0 \mathrm{~J}$ for DOS was used for coding, which simplified the connection with the assembly language, which had become complicated when BASIC was used. In order to avoid I/O interruption and carry out safe time measurement, care was taken with the programming to access the recording devices after the session (Funakawa, 1988, p. 82). For coding, Adachi (1995) and Nakashima (1997) were also referred to, as well as Graves and Bradley (1987).

\section{Preliminary Step 1B: Precision Evaluation of the PC Clock}

Equipment. The constructed program was executed on an AT-compatible Fujitsu FMV-5166T3 computer with an FMV-KB321 keyboard. The operating system was DOS, Version 6.2/V. An Iwatsu Electric Company SC-7203 UC was used to evaluate the time measurement value obtained from the PC.

Accuracy evaluation. The verification of whether accurate time measurement was actually possible was made by measuring the vertical synchronous signal interval of the monitor display. In order to examine accurately the frequency time for one frame of the display, a photodiode sensor was attached to the front of the monitor, and a fre-

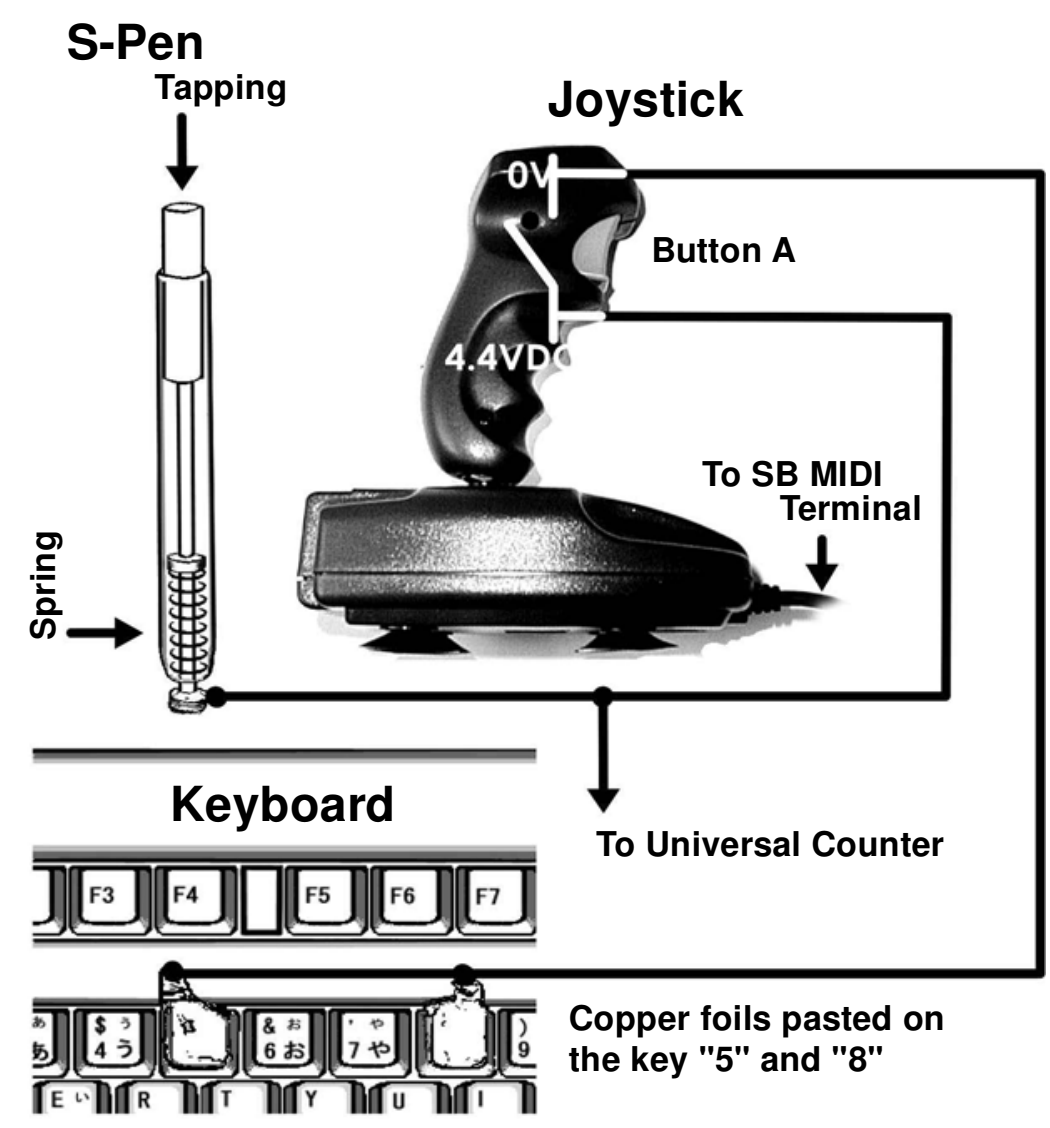

Figure 1. Connection diagram of a joystick, pen with a built-in spring (S-Pen), and keyboard. 
Table 1

Sets of PC, Keyboard, and MIDI Boards Investigated: The Mean and Standard Deviation of the Subtraction Value of the Corresponding UC's Value From the Timing Value of the Joystick

\begin{tabular}{clllll}
\hline PC Model & \multicolumn{1}{c}{ CPU (Speed) } & PCI MIDI Board & \multicolumn{1}{c}{ Keyboard Model } & $M$ & $S D$ \\
\hline Fujitsu FMV & & & & & \\
5166T3 & Pentium (166 MHz) & Creative SB AWE32 & FMV KB321 (PS/2) & 0.009 & 0.093 \\
TV337 & Pentium II (333 MHz) & Creative SB AWE64 & FMV KB321 (PS/2) & 0.117 & 0.071 \\
M3/557 & Athlon (550 MHz) & Yamaha YMF744B & CP018203-02(USB) & 0.134 & 0.062 \\
& & & IBM 5576-B01 (PS/2) & 0.036 & 0.099 \\
Dell Dimension & & & & & \\
XPS B800r & Pentium III (800 MHz) & Creative SB Live & SK-800 (PS/2) & 0.053 & 0.084 \\
4100 & Pentium III (800 MHz) & Creative SB Live & SK-800 (PS/2) & 0.056 & 0.137 \\
8100 & Pentium 4 (1.5 GHz) & Creative SB Live & RT7D00 (PS/2) & 0.119 & 0.073 \\
\hline
\end{tabular}

Note-Means and Standard deviations are measured in milliseconds.

quency of $59.662 \mathrm{~Hz}$ was obtained by feeding the output to the UC. The periodic interval was $16.76109 \mathrm{msec}$. The data obtained from the PC had three values of 16.76106 (the mode), 16.76190, and $16.76023 \mathrm{msec}$, with a range of $\pm 0.84 \mu \mathrm{sec}$. This agrees with the theoretically estimated error.

\section{Preliminary Step 2: Time Elapsed From Key Touch to Key-On}

The next problem to be solved was how to keep the elapsed time from key contact to key-on as constant as possible, and so a simple pen shape with a built-in spring (S-Pen; Figure 1) was made to enable the key to be tapped accurately at high speed. A key-tapping experiment was carried out separately with this S-Pen on another FMVK321 keyboard. A circuit consisting of a resistor and a battery was made by using copper foil attached to the surface of the key and the tip of the S-Pen, which were used as switching terminals that would cause a voltage change when they came into contact. A similar simple circuit consisting of a resistor and a battery was also constructed so that a voltage change would be produced when the key input became effective (key-on). By using these two voltage changes as start and stop signals for the UC, it was possible to examine the time elapsed from key contact until effective key input. Figure 2 shows the elapsed time from 500 trials as a frequency distribution $(M=2.007$ msec, $S D=0.259 \mathrm{msec}$ ).

\section{VERIFICATION OF THE RELIABILITY OF TIME MEASUREMENT BY A PC'S CLOCK AND JOYSTICK}

How closely does the timing value of the PC's clock and joystick agree with the value of an external UC when one measures the time from speaker-on to joystick-on? The PC's timing value is the difference of the PC's two real times that correspond to the two signals, respectively. The UC's timing value is obtained by starting the UC with a voltage change of the speaker terminal and stopping it with a joystick-on. Keeping in mind the next main objective, the identification of the keyboard response characteristics, the wiring was arranged so that the three signals, joystick-on, UC-stop, and key-on, were simultaneously generated by one keypress.

\section{Details of Equipment and Wiring}

The attachment of a lead to the speaker terminal to start the UC. In order to confirm the general applicability of this method, an investigation was carried out on the six computers listed in Table 1. A hole was made in the steel plate at the back of the motherboard of five PCs, which corresponded to the speaker position, and then the lead was attached through this hole to the speaker terminal. (A method for converting the speaker sound into an electric signal with a microphone was also considered but was rejected because of a conversion time of about $1.5 \mathrm{msec}$ ).

The construction of the bypass for joystick button $A$. The joystick used here was the JSK-104 made by the Try Corporation with a D-Sub15 game pin correspondence (any 15-pin-compatible model is usable). We connected one lead wire to the S-Pen and one contact point of the joystick button A. Another lead wire was attached to the other contact point of the button, and then two copper foils in series were connected to its other side. Each foil was

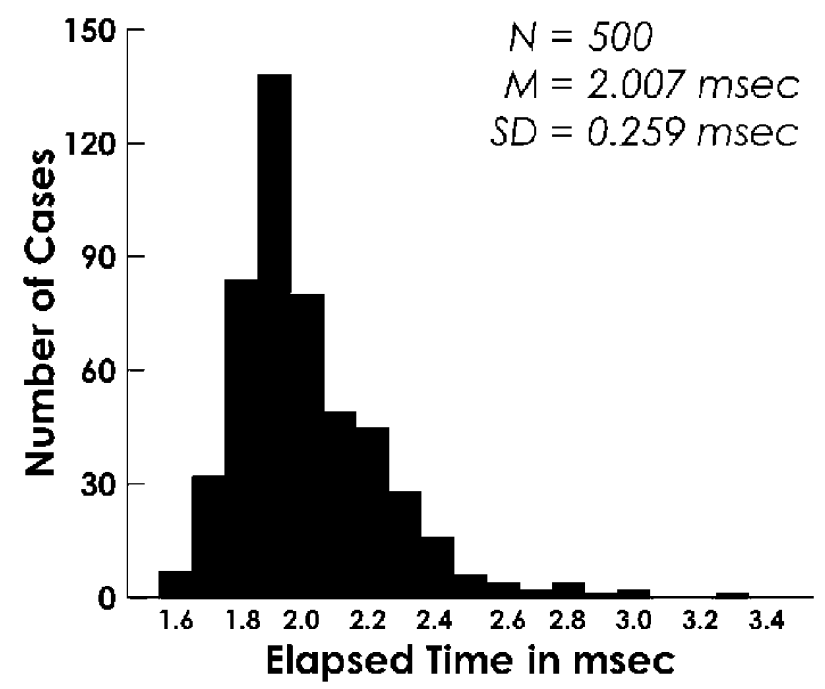

Figure 2. Time elapsed from key contact to key-on using an S-Pen. 
Table 2

The Mean and Standard Deviation of the Difference of Keyboard and Joystick Data and the Estimated Value of the Keyboard Scan Interval Time from the Scan Interval Under RT Conditions

\begin{tabular}{|c|c|c|c|c|c|c|c|}
\hline PC Model & CPU (Speed) & $\begin{array}{c}\text { Keyboard } \\
\text { Model }\end{array}$ & $\begin{array}{c}\text { Keyboard Scan } \\
\text { Frequency }\end{array}$ & $\begin{array}{c}\text { Scan Interval } \\
\text { Time }\end{array}$ & $M$ & $S D$ & $\begin{array}{c}\text { Estimated Scan } \\
\text { Interval Time } \\
\end{array}$ \\
\hline \multicolumn{8}{|l|}{ Fujitsu FMV } \\
\hline \multirow[t]{2}{*}{$5166 \mathrm{~T} 3$} & Pentium (166 MHz) & FMV KB321 (PS/2) & $171.6 \mathrm{~Hz}$ & 5.83 & 15.018 & 1.689 & 5.85 \\
\hline & & IBM 5576-B01 (PS/2) & $97.1 \mathrm{~Hz}$ & 10.30 & 23.485 & 2.911 & 10.08 \\
\hline TV337 & Pentium II (333 MHz) & FMV KB321 (PS/2) & $171.6 \mathrm{~Hz}$ & 5.83 & 15.794 & 1.694 & 5.87 \\
\hline \multirow[t]{6}{*}{ M3/557 } & Athlon (550 MHz) & CP018203-02 (USB) & $100.4 \mathrm{~Hz}$ & 9.96 & 72.936 & 5.419 & $18.77 *$ \\
\hline & & Mets CK-006 (USB) & $244.2 \mathrm{~Hz}$ & 4.10 & 27.553 & 9.453 & $32.75^{*}$ \\
\hline & & Mitsumi KPQ-EA9EA (PS/2) & $87.4 \mathrm{~Hz}$ & 11.44 & 29.614 & 3.140 & 10.88 \\
\hline & & RT7D00 (PS/2) & $338.1 \mathrm{~Hz}$ & 2.96 & 23.046 & 0.873 & 3.02 \\
\hline & & SK-800 (PS/2) & $233.8 \mathrm{~Hz}$ & 4.28 & 12.433 & 1.215 & 4.21 \\
\hline & & IBM 5576-B01 (PS/2) & $97.1 \mathrm{~Hz}$ & 10.30 & 24.755 & 2.937 & 10.17 \\
\hline \multicolumn{8}{|c|}{ Dell Dimension } \\
\hline XPS B800 & Pentium III (800 MHz) & SK-800 (PS/2) & $233.8 \mathrm{~Hz}$ & 4.28 & 10.785 & 1.211 & 4.20 \\
\hline 4100 & Pentium III (800 MHz) & SK-800 (PS/2) & $233.8 \mathrm{~Hz}$ & 4.28 & 10.816 & 1.204 & 4.17 \\
\hline \multirow[t]{3}{*}{8100} & Pentium $4(1.5 \mathrm{GHz})$ & RT7D00 (PS/2) & $338.1 \mathrm{~Hz}$ & 2.96 & 21.230 & 0.836 & 2.90 \\
\hline & & SK-800 (PS/2) & $233.8 \mathrm{~Hz}$ & 4.28 & 10.614 & 1.221 & 4.23 \\
\hline & & IBM 5576-B01 (PS/2) & $97.1 \mathrm{~Hz}$ & 10.30 & 23.245 & 2.951 & 10.22 \\
\hline
\end{tabular}

Note-All times, means, and standard deviations are measured in milliseconds. *Notice USB keyboard.

fixed, respectively, on the surface of the target key " 5 " and the dummy key " 8 " (Figure 1). Thus, striking a foil-fixed key with the S-Pen resulted in generating a joystick-on signal and a UC-stop signal simultaneously and in generating a key-on signal after a physical delay time of a few milliseconds.

The connection of a joystick to a MIDI board. MIDI boards with PCI connections for DOS were required and are listed in Table 1. A joystick was connected to each one.

The UC and other equipment. The UC used at Preliminary Step 1B was used again to check the timing data recorded by the joystick. A GP-IB board SC-0111 was connected to an NEC PC9821 Ap to collect UC data automatically. The monitoring of the UC input waveform was carried out on a Hitachi Oscilloscope V-550.

\section{Procedure}

To compare the PC's timing data from the joystick, the UC was started by the PC speaker terminal voltage change in the PC (following Graves \& Bradley, 1987) and was stopped by the voltage change (drop from $4.4 \mathrm{~V}$ [DC] to $0 \mathrm{~V}$ ) caused by contact of the " 5 " key and the S-Pen. Trials of striking the " 5 " key after the sound were carried out 100 times, makes up one trial block. After five trial blocks, 500 matched pairs of UC data and PC data were obtained. After the procedure, we collected data from six sets of PCs and keyboards and also from a PC (FMV M3/557) connected with an IBM PS/2 keyboard. The PCs and keyboards investigated here are listed in Table 1.

\section{Results and Discussion}

The validity of the use of the joystick was greatest when the difference between the timing values from the joystick and from the UC was the smallest. The differences between the 500 matched pairs of joystick values and the UC data were examined, and then the mean and standard deviation for each keyboard were calculated (Table 1). The means and standard deviations were about $0.1 \mathrm{msec}$, as is

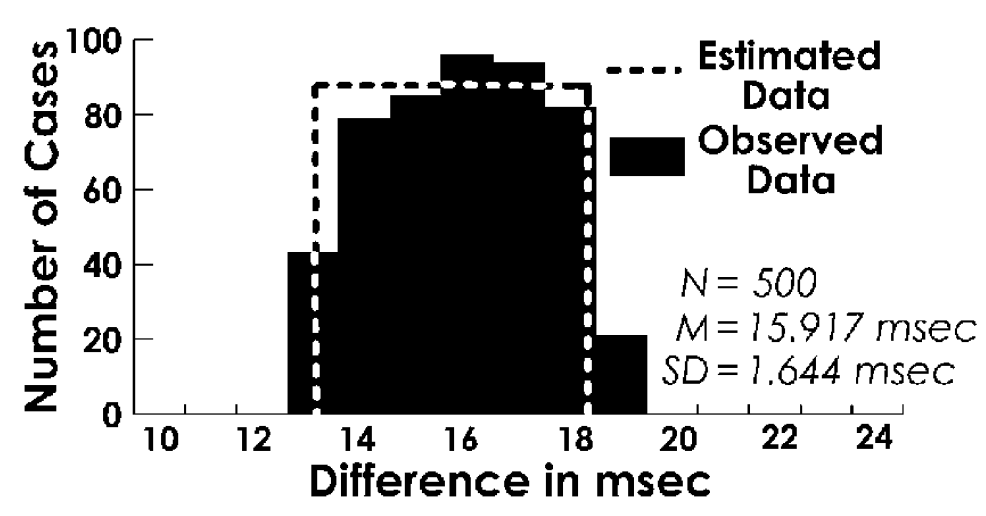

Figure 3. The frequency distribution of the differences in response times measured with a keyboard and a joystick using an FM V-5166T3 (Pentium 166 MHz CPU) and FMV KB321 keyboard. 
Table 3

The Different Values of Joystick and Keyboard Data and Their Mean and Standard Deviations Under the IRT Condition

\begin{tabular}{|c|c|c|c|c|c|c|c|}
\hline PC Model & CPU (Speed) & $\begin{array}{c}\text { Keyboard } \\
\text { Model }\end{array}$ & $\begin{array}{c}\text { Keyboard Scan } \\
\text { Frequency }\end{array}$ & $\begin{array}{c}\text { Scan Interval } \\
\text { Time }\end{array}$ & $M$ & $S D$ & $\begin{array}{c}\text { Estimated Scan } \\
\text { Interval Time }\end{array}$ \\
\hline \multicolumn{8}{|l|}{ Fujitsu FMV } \\
\hline $5166 \mathrm{~T} 3$ & Pentium (166 MHz) & FMV KB321 (PS/2) & $171.6 \mathrm{~Hz}$ & 5.83 & -0.003 & 2.553 & 6.25 \\
\hline TV337 & Pentium II (333 MHz) & FMV KB321 (PS/2) & $171.6 \mathrm{~Hz}$ & 5.83 & -0.306 & 2.599 & 6.37 \\
\hline \multirow[t]{2}{*}{ M3/557 } & Athlon $(550 \mathrm{MHz})$ & CP018203-02 (USB) & $100.4 \mathrm{~Hz}$ & 9.96 & -0.254 & 7.930 & $19.43 *$ \\
\hline & & IBM 5576-B01 (PS/2) & $97.1 \mathrm{~Hz}$ & 10.30 & -0.026 & 4.133 & 10.12 \\
\hline \multicolumn{8}{|l|}{ Dell Dimension } \\
\hline XPS B800 & Pentium III (800 MHz) & SK-800 (PS/2) & $233.8 \mathrm{~Hz}$ & 4.28 & 0.026 & 1.760 & 4.31 \\
\hline 4100 & Pentium III (800 MHz) & SK-800 (PS/2) & $233.8 \mathrm{~Hz}$ & 4.28 & -0.006 & 1.666 & 4.07 \\
\hline 8100 & Pentium 4 (1.5 GHz) & RT7D00 (PS/2) & $338.1 \mathrm{~Hz}$ & 2.96 & 0.001 & 1.156 & 2.83 \\
\hline
\end{tabular}

Note-The keyboard scan interval time was estimated from the standard deviation. All times, means, and standard deviations are measured in milliseconds. *Notice USB keyboard.

shown in Table 1. Given this agreement, the joystick can be used as a replacement for the UC.

\section{IDENTIFICATION OF KEYBOARD RESPONSE CHARACTERISTICS OF JOYSTICKS UNDER RT CONDITIONS}

When the time interval from the speaker-on to the keyon is measured by the keyboard, its interval data contains the total mean delay time and the variance by the keyboard scanning, as described above. These keyboard response characteristics were clarified in the comparison with the joystick data. In particular, the subtraction values of joystick data values from keyboard data values were analyzed.

\section{Equipment and Procedure}

The equipment and procedure were the same as those described above in the verification of the reliability of using the joystick. However, in order to clarify the relationship between the PC and the keyboard, keeping the abovementioned six PCs intact, new keyboards were added, and exchanges of keyboards and PCs were tried out.

\section{Results and Discussion for the RT Condition}

Five hundred matched pairs of joystick and keyboard data were obtained for each keyboard. The means and standard deviations were calculated for the difference between the joystick and the keyboard data values, and the results are shown in Table 2.

The theory and practice for recording the keyboard scan frequency in Table 2 are as follows. A scanning pulse appears at key contact points when a key is pressed. Therefore, if a key is continually pressed, a series of pulses will be recorded. The period of these pulses gives the keyboard scan frequency. All keyboards investigated, except for the IBM keyboard, were composed of two printed sheets and one separate sheet that had many round clipped holes to enable a key-on when pressed. Therefore, a solder ball formed at the tip of a lead wire was inserted between the printed sheets in order to keep the key contact points at the short state, and then the other side of the wire was connected to the UC.

If the keyboard error variance was caused mainly by keyboard scanning, the keyboard scan interval time $(1,000$ msec/[keyboard Scan Frequency]) could be estimated from the $S D$ of the difference data. The distribution of the difference value by the keyboard scanning was assumed theoretically to be a uniform distribution, whereas the distribution of the actual data also seemed to be a uniform distribution (Figure 3). Therefore, the $S D_{\mathrm{rt}}$ of the data gives the estimation value of the keyboard scan interval by $R_{\mathrm{rt}}^{2}=S D_{\mathrm{rt}}^{2} \times 12$ (Hayashi, $1985, \mathrm{p} .160 ; R_{\mathrm{rt}}$ means the

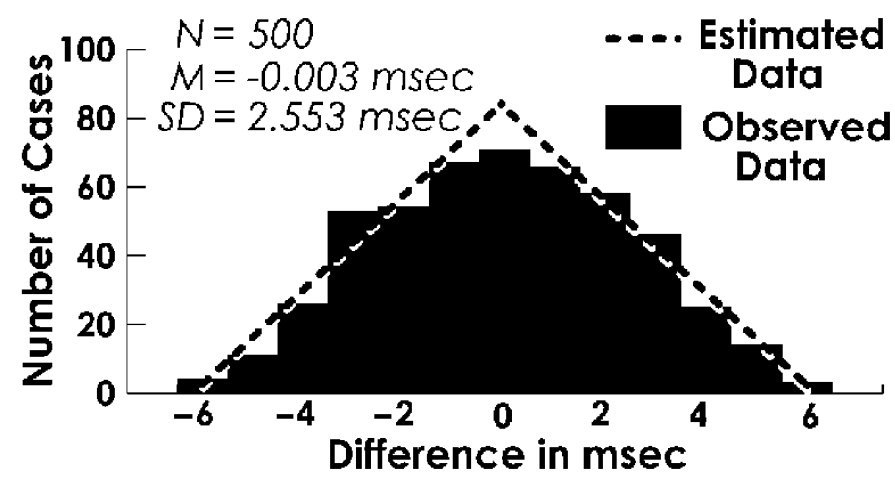

Figure 4. The frequency distribution of the differences in interresponse time measured with a keyboard and a joystick, using an FMV5166 T3 (Pentium $166 \mathrm{MHz}$ CPU) and FMV KB321 keyboard. 
range of the uniform distribution, a keyboard scan interval time). The estimated scan interval time from each of the PS/2 keyboards' data (except for two USB keyboards' data) agrees largely with the value measured directly from the keyboard circuit, as is shown in Table 2.

As was described above, the total mean delay time of a PS/2 keyboard consisted of the keyboard physical delay time, the delay time by keyboard scanning, and the fixed delay time for converting the key-on signal to character information and transmitting it to the CPU. To find the fixed delay time, we can subtract the $2 \mathrm{msec}$ of the physical delay time and half of the keyboard scan interval time from the total mean delay time. However, it may be sufficient to know the keyboard total delay time from the viewpoint of the data correction. The data correction will actually be carried out by subtracting this total mean delay time from the recorded RT.

\section{IDENTIFICATION OF THE KEYBOARD RESPONSE CHARACTERISTICS OF JOYSTICKS UNDE R IRT CONDITIONS}

How much do keyboard and joystick data differ in measuring the time interval of successive key touches? As is clear from the data under the RT condition, the real time recorded at one key touch always shows the lag of the total mean delay time accompanied by the keyboard error variance. The real time recorded at the next key touch also shows the delay time with the variance. Therefore, it is assumed that the mean is zero and that only the two-fold variance remains as the difference between the keyboard data and the joystick data under the IRT condition. Since the error distribution under the RT condition is a uniform distribution, the distribution under the IRT condition becomes a triangular distribution. This fact was confirmed in the time measurement via the joystick.

\section{Equipment and Procedure}

The equipment and procedure were the same as those for the RT conditions. However, in the IRT condition, the " 8 " key was struck 101 times consecutively with the S-Pen after the sound was made, and then the " 5 " key was struck. This constituted one block, and 500 measured values were obtained in five blocks (the initial response was excluded because it corresponded to the RT reaction). The investigation of the IRT condition was carried out with the six PCs and seven keyboards used in the verification of the reliability of the joystick.

\section{Results and Discussion for the IRT Condition}

We calculated the difference between joystick and keyboard data in the IRT condition, and also its mean and standard deviation. The mean value of the difference is estimated to be close to zero, as is shown in Table 3. The difference distribution also is triangular, as is shown in Fig- ure 4, which was obtained by a PC FMV5166T3. From $S D_{\text {irt }}$, the keyboard scanning interval time $\left(R_{\mathrm{rt}}\right)$ can be estimated using the equations $R_{\mathrm{irt}}^{2}=S D_{\mathrm{irt}}^{2} \times 24$ and $R_{\mathrm{rt}}=$ $R_{\text {irt }} / 2$.

\section{CONCLUSION}

A joystick connected to a MIDI board clarifies the keyboard total mean delay time and the error variance when we use a keyboard as a response panel, instead of relying on an external UC. This provides us with an alternative method of carrying out the recommendation of Segalowitz and Graves (1990).

Although we attempted to collect data under both RT and IRT conditions in this paper, the RT data are more than sufficient to identify keyboard response characteristics, since the RT data give an estimation of the IRT error variance, in addition to the keyboard total mean delay time.

\section{REFERENCES}

ADACHI, T. (1995). DOS/V programming reference [in Japanese]. Tokyo: Softbank.

FAY, D. Q. M. (2000). Peripherals. http//www.rz.uni-hohenheim. de/sys/basics/csc102/ ch25.html.

FUNAKAWA, M. (1988). Control technique in experiment and subroutine programs written in Machine Language (PET) [in Japanese]. In J. Abe (Ed.), Programming for psychological experiments using PCs (pp. 73212). Tokyo: Brain.

GRAVES, R., \& BRADLEy, R. (1987). Millisecond interval timer and auditory reaction time programs for the IBM PC. Behavior Research Methods, Instruments, \& Computers, 19, 30-35.

HayAshi, C. (1985). Probability theory and statistics [in Japanese]. Tokyo: NHK.

Kawamura, K., \& Yoshida, M. (1995). Architecture handbookfor AT compatible PCs [in Japanese]. Tokyo: Natsume.

NAKASHIMA, N. (1997). An introduction to I/O control programming for $C \& C++$ programmers [in Japanese]. Tokyo: CQ.

PASHLER, H. (1994). Overlapping mental operations in serial performance with preview. Quarterly Journal of Experimental Psychology, 47A, 161-191.

Segalowitz, S. J., \& Graves, R. E. (1990). Suitability of the IBM XT, AT, and PS/2 keyboard, mouse, and game port as response devices in reaction time paradigms. Behavior Research Methods, Instruments, \& Computers, 22, 283-289.

Smith, B., \& Puckett, T. (1984, April). Life in the fast lane. PCTech Journal, pp. 63-74.

UlRICH, R., \& GIRAY, M. (1989). Time resolution of clocks: Effects on reaction time measurement-Good news for bad clocks. British Journal of Mathematical \& Statistical Psychology, 42, 1-12.

Verwey, W. B., \& Dronkert, Y. (1996). Practicing a structured continuous key-pressing task: Motor chunking or rhythm consolidation? Journal of Motor Behavior, 28, 71-79.

\section{NOTE}

1. The source program and executable files mentioned in this article are downloadable from http://www.dmedia.smedia.aichi-edu.ac.jp/.

(Manuscript received March 30, 2001; revision accepted for publication January 3, 2002.) 